Pacific Journal of Mathematics

RATIONAL SURGERY CALCULUS: EXTENSION OF KIRBY'S 


\title{
RATIONAL SURGERY CALCULUS: EXTENSION OF KIRBY'S THEOREM
}

\author{
DALE RolfSEN
}

Every 3-dimensional closed orientable manifold may be constructed by surgery on the 3-sphere as introduced by M. Dehn. We are concerned in this paper with answering the question: when do two surgery schemes actually produce the same 3-manifold?

Kirby [4] described certain geometric moves - a calculus of links and showed that two "framed links" in $S^{3}$ produce homeomorphic surgery manifolds if and only if they are related by these moves. But not all surgeries are permitted in the Kirby calculus. Only those which correspond to attaching 2-handles to the 4-ball are considered, since Kirby's techniques are 4-dimensional.

EXAMPLE. Dehn considered the homology spheres determined by the surgery data

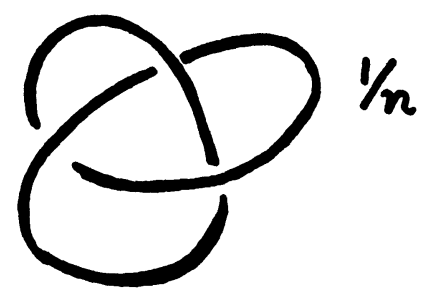

where $n$ is an integer (notation of [6], also explained below.) Except for $n= \pm 1$, these constructions are not considered in the Kirby calculus. In continuations of this example we will show that these spaces are all distinct. One can, of course, construct these manifolds using surgeries permitted in the Kirby calculus, but one is obliged to use different knots $K_{n}$ for each $n$, which we will construct.

In this paper we prove a generalization of Kirby's theorem which applies to the entire class of Dehn surgeries on $S^{3}$. Instead of Kirby's calculus, we use the "rational" link calculus introduced (independently) in [6]. Our main result states that different surgery data (= links with rational coefficients) yield the same oriented manifold if and only if the 
data are related by a finite sequence of moves of the calculus of [6]. The proof depends on Kirby's theorem, and on a theorem of Rourke and Fenn which provides the essential connection between the calculi [2].

Surgery data and their modification. The rational surgery calculus deals with surgery data $(V, J)$, where $V=V_{1} \cup \cdots \cup V_{n}$ is a disjoint collection of solid tori $\left(V_{i} \cong S^{1} \times D^{2}\right)$ in $S^{3}$ and $J=J_{1} \cup \cdots \cup J_{n}$ a collection of Jordan curves $J_{i} \subset \partial V_{i}$. We require only that $J_{i}$ be essential (= nonseparating) in the torus $\partial V_{i}$. The Kirby calculus requires also that $J_{i}$ be a longitude (i.e. homologous with the centerline in $V_{i}$ ). If every $J_{i}$ is a longitude, we say that $(V, J)$ is integral.

The surgery manifold is the space

$$
M(V, J)=S^{3}-\stackrel{\circ}{V}+V
$$

obtained by removing the interior of the solid tori and sewing them back with any attaching homeomorphism

$$
h: \partial V \rightarrow \partial\left(S^{3}-\stackrel{\circ}{V}\right)=\partial V \text { such that } h\left(\text { meridian of } V_{i}\right)=J_{i}
$$

"Meridian" means the curve $* \times S^{1}$ in $S^{1} \times D^{2}$.

Then $M(V, J)$ is a well-defined closed 3-manifold, with a naturally preferred orientation, agreeing with the orientation for $S^{3}$, which we take as fixed throughout the discussion.

We now consider three ways of modifying surgery data $(V, J)$ to obtain the data $\left(V^{\prime}, J^{\prime}\right)$.

(I) Homeomorphism. Let $h: S^{3} \rightarrow S^{3}$ be an orientation preserving homeomorphism and define $V^{\prime}=h(V), J^{\prime}=h(J)$.

(II) Twist. Locate (if possible) an unknotted component, say $V_{1}$ of $V=V_{1} \cup \cdots \cup V_{n}$. Then there is a thickened disk $D^{2} \times I$ in $S^{3}-\stackrel{\circ}{1}_{1}$ which is attached to $\partial V_{1}$ along $S^{1} \times I$. The self-homeomorphism $(x, t) \rightarrow$ $\left(x e^{2 \pi i t}, t\right)$ of $D^{2} \times I$ (in $\mathrm{C} \times I$ ), extended by the identity off of $D^{2} \times I$, defines a "twist homeomorphism"

$$
g: S^{3}-\stackrel{\circ}{V}_{1} \rightarrow S^{3}-\stackrel{\circ}{V}_{1}
$$

let

$$
V^{\prime}=V_{1} \cup g V_{2} \cup \cdots \cup g V_{n}, \quad J^{\prime}=g J_{1} \cup g J_{2} \cup \cdots \cup g J_{n} .
$$

(III) Trivial insertion (deletion). Given $V=V_{1} \cup \cdots \cup V_{n}$, let $V_{n+1}$ by any solid torus disjoint from $V$ and let $J_{n+1} \subset \partial V_{n+1}$ be a meridian of 
$V_{n+1}$. Define

$$
\left(V^{\prime}, J^{\prime}\right)=\left(V \cup V_{n+1}, J \cup J_{n+1}\right) .
$$

This is a trivial insertion. Its inverse is a trivial deletion, i.e. delete any $V_{l}$ for which $J_{i}$ is a meridian.

LEMMA 1. If $\left(V^{\prime}, J^{\prime}\right)$ results from $(V, J)$ by a move of types I, II or III, then $M(V, J)$ and $M\left(V^{\prime}, J^{\prime}\right)$ are orientation-preserving homeomorphic.

The proof is completely routine. In brief, here are the two theories:

Rational Calculus.

Objects are surgery data $(V, J)$.

Equivalence is the relation " $\leftrightarrow$ " generated by (I), (II), (III).

Kirby Calculus.

Objects are integral surgery data.

Equivalence is the relation “ $\underset{\partial}{\approx}$ " generated by the Kirby moves [4].

Statement of results. Let $\cong$ mean “orientation-preserving homeomorphic".

TheOrem 1. Let $(V, J)$ and $\left(V^{\prime}, J^{\prime}\right)$ be surgery data in $S^{3}:$ Then

$$
M(V, J) \cong M\left(V^{\prime}, J^{\prime}\right) \text { if and only if }(V, J) \leftrightarrow\left(V^{\prime}, J^{\prime}\right) .
$$

Lemma 1 proves the easy direction. To prove the converse we need three results.

Kirby's TheOREM [4]. If $(V, J)$ and $\left(V^{\prime}, J^{\prime}\right)$ are integral surgery data in $S^{3}$, then

$$
M(V, J) \cong M\left(V^{\prime}, J^{\prime}\right) \text { if and only if }(V, J) \underset{\partial}{\approx}\left(V^{\prime}, J^{\prime}\right) .
$$

LEMMA 2. (Fenn-Rourke [2], or see [4].) If $(V, J)$ and $\left(V^{\prime}, J^{\prime}\right)$ are integral, then

$$
(V, J) \underset{\partial}{\approx}\left(V^{\prime}, J^{\prime}\right) \quad \text { implies } \quad(V, J) \leftrightarrow\left(V^{\prime}, J^{\prime}\right) .
$$

LEMMA 3. Given surgery data $(V, J)$, there exist integral surgery data $\left(V_{I}, J_{I}\right)$ such that $(V, J) \leftrightarrow\left(V_{I}, J_{I}\right)$. 
To complete the proof of Theorem 1 , assume $M(V, J) \cong M\left(V^{\prime}, J^{\prime}\right)$. Then by Lemma 3 , there are integral data with $(V, J) \leftrightarrow\left(V_{I}, J_{I}\right)$ and $\left(V^{\prime}, J^{\prime}\right) \leftrightarrow\left(V_{I}^{\prime}, J_{I}^{\prime}\right)$. By Lemma $1, M\left(V_{I}, J_{I}\right) \cong M\left(V_{I}^{\prime}, J_{I}^{\prime}\right)$, and by Kirby and Lemma 2, we conclude that $\left(V_{I}, J_{I}\right) \leftrightarrow\left(V_{I}^{\prime}, J_{I}^{\prime}\right)$. It follows that $(V, J)$ $\leftrightarrow\left(V^{\prime}, J^{\prime}\right)$ and Theorem 1 is proved.

COROLlaRY. Kirby's calculus equals the rational calculus, restricted to integral surgery data. That is, if $(V, J)$ and $\left(V^{\prime}, J^{\prime}\right)$ are integral then the following are equivalent:

(a) $(V, J) \underset{\partial}{\approx}\left(V^{\prime}, J^{\prime}\right)$

(b) $(V, J) \leftrightarrow\left(V^{\prime}, J^{\prime}\right)$;

(c) $M(V, J) \cong M\left(V^{\prime}, J^{\prime}\right)$.

To prove Lemma 3, it will be very useful to formulate surgery data in terms of links with rational labels, as in [6]. The surgery data $(V, J)$ is described by the link $L=L_{1} \cup \cdots \cup L_{n}$, where $L_{i}$ is a central curve $\left(S^{1} \times *\right.$ in $\left.S^{1} \times D^{2}\right)$ of $V_{i}$. The attachment curve $J_{i} \subset \partial V_{i}$ is completely determined up to isotopy by the fraction

$$
r_{i}=p_{i} / q_{i} \quad\left(=\infty \text { if } q_{i}=0\right)
$$

where

$$
\left[J_{i}\right]=q_{i}\left[L_{l}\right] \quad \text { in } H_{1}\left(V_{i} ; \mathbf{Z}\right) \quad \text { and } \quad p_{i}=l k\left(J_{i}, L_{i}\right)
$$

Although one must orient $J_{i}$ and $L_{i}$ for this to make sense, the ratio $r_{i}$ is independent of such choice. The choice of a sign convention for computing linking number " $l k$ " is tantamount to a choice of preferred orientation of $S^{3}$. We will use the convention under which the following has linking number +1 .

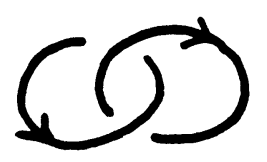

In this setting the moves of the rational surgery calculus revise a labelled link as follows:

(I) Replace $L$ by a link $L^{\prime}$ of same (oriented) link type in $S^{3}$. Keep the same coefficients. 
(II) Replace $L$ by $L^{\prime}$ as shown:
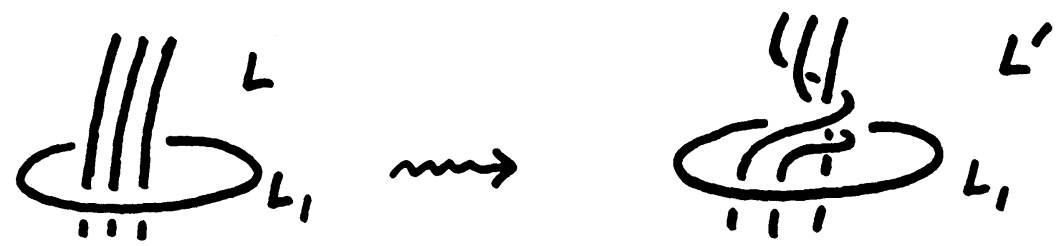

The new coefficients $r_{i}^{\prime}$ are defined:

$$
\begin{cases}r_{1}^{\prime}=\frac{1}{1 / r_{1}+\tau} & (\tau=\text { number of right-hand twists }), \\ r_{i}^{\prime}=r_{i}+\tau\left[l k\left(L_{i}, L_{1}\right)\right]^{2} & (i \neq 1) .\end{cases}
$$

(III) Introduce (or delete) a component of the link with coefficient $\infty$.

We point out that $(V, J)$ is integral if and only if each $r_{i}$ is an integer (finite). In this case $r_{i}$ is exactly the "framing index" of Kirby [4].

EXAMPLE (continued). We will use the rational calculus to show that

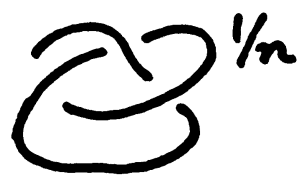

the homology spheres resulting from surgery on the trefoil are Seifert fibre spaces. The following pictures all describe the same manifold.

Trivial insertion

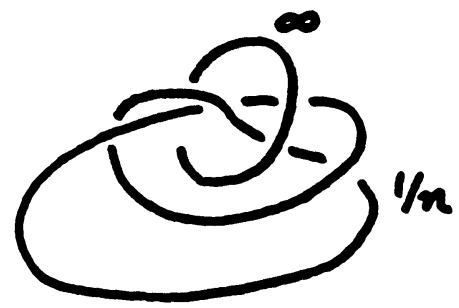

Twist $(\tau=-1)$

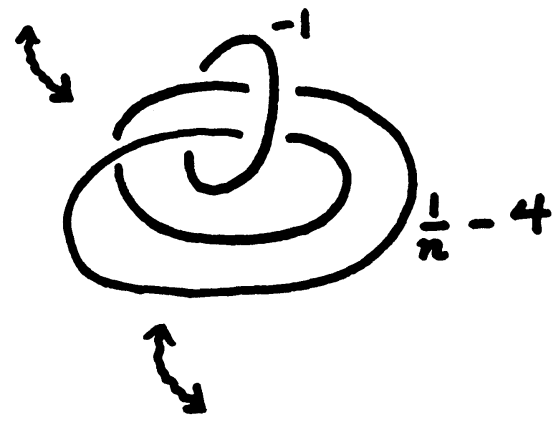


Homeomorphism and trivial insertion

Twist $(\tau=-2)$

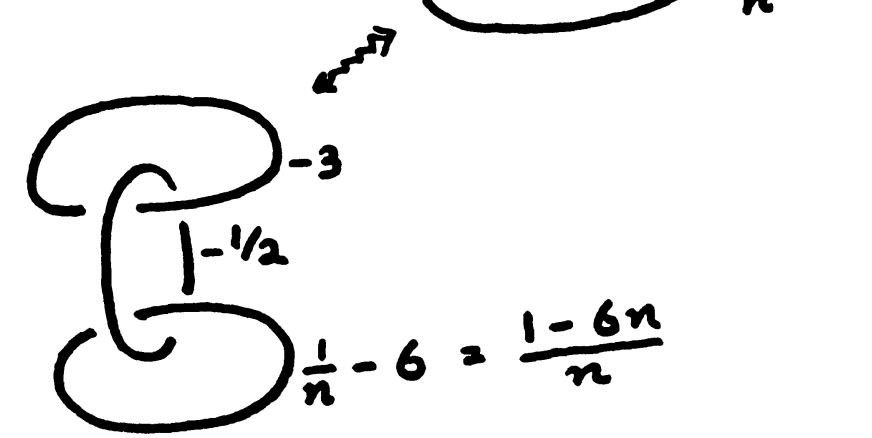

The $-\frac{1}{2}$ curve has complement fibred by circles, with the other curves as fibres. Using this one obtains a fibration of the surgery manifold with three singular fibres, of multiplicities 2,3 and $|1-6 n|$. (In case $n=0$, the surgery manifold is $S^{3}$ and there are just two singular fibres.) According to Seifert [7], Satz 12, each homology sphere (except $S^{3}$ ) has a unique Seifert fibring. It follows that the manifolds constructed above are distinct for different $n$.

Proof of Lemma 3. Given $(V, J)$, consider the associated labelled link $(L, r)=\left(L_{1} \cup \cdots \cup L_{n} ; r_{1}, \ldots, r_{n}\right)$. Using deletions, we may assume each $r_{i}$ is finite. Write $r_{i}=p_{i} / q_{i}$ with $q_{i}>0$. If each $q_{i}=1$ we are done. Otherwise, suppose some component, $L_{1}$ say, has label $r_{1}=p / q, q \geq 2$. Perform the following modifications on $L$ :

Step 1. Unknot $L_{1}$. If one considers any "regular projection" of $L_{1}$, one may unknot $L_{1}$ by changing some crossings. This may be effected (following Hempel [3]) by insertions and twists thus:
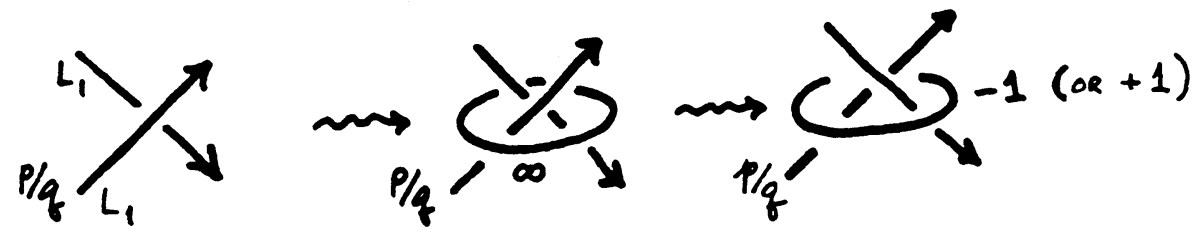

Step 2. By the following operation, we may revise $p$ modulo $q$ and assume $0<p<q$. 

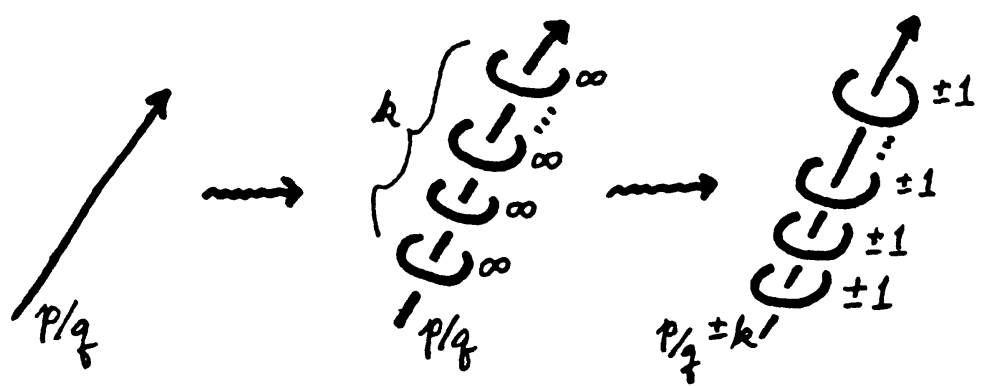

Step 3. Twist about $L_{1}$ with a left-hand twist. Then the coefficient $r_{1}=p / q(0<p<q)$ becomes

$$
r_{1}^{\prime}=\frac{1}{q / p-1}=\frac{p}{q-p} .
$$

Note that $0<q-p<q$.

Thus for any component, we can reduce the denominator by insertions and twists. In the process, no new fractional labels have been introduced and the old fractional labels of components $L_{2}, \ldots, L_{\mu}$ are only changed by an integer. So inductively one may reduce all denominators to +1 and the lemma follows.

EXAMPLE (concluded). Here is a sequence which shows that the homology spheres constructed by $1 / n$ surgery on the trefoil may be obtained by +1 surgery on some other knot (depending on $n$, of course).
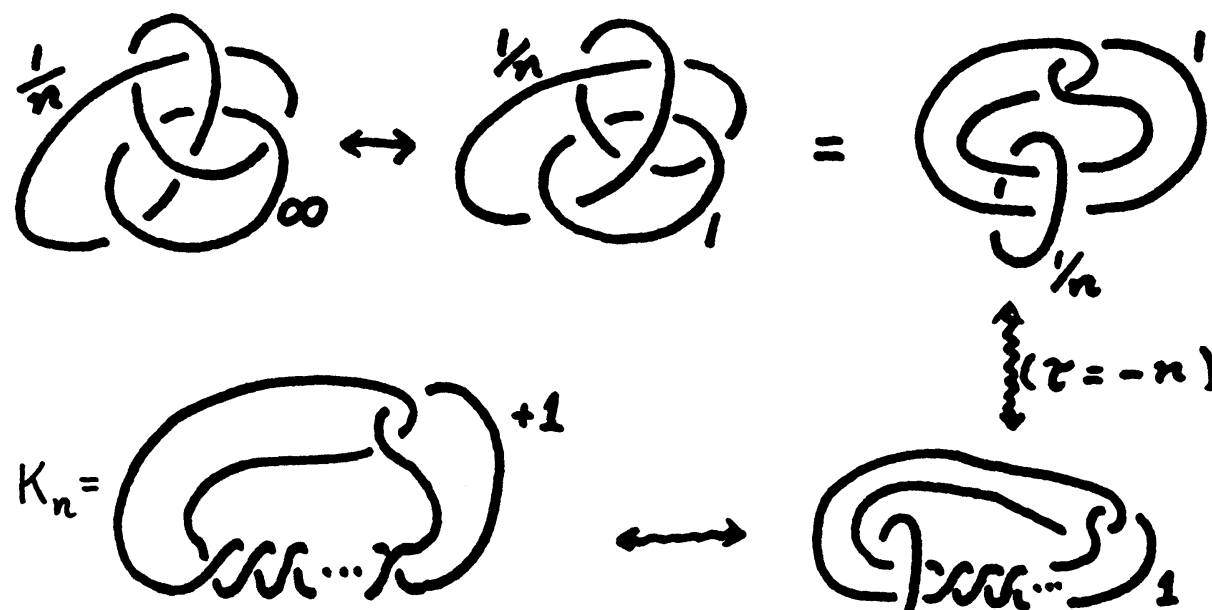

( $n$ full left-hand twists)
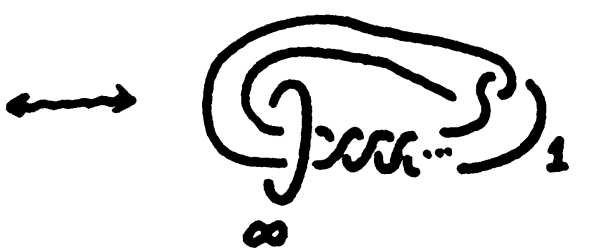

Note that $K_{1}$ is just the trefoil again! $K_{-1}$ is the figure 8 knot. 
An application. Theorem 1 suggests possibilities for new tools for the study of 3-manifolds, coming from knot and link theory. Indeed any algebraic invariant of links (with coefficients) automatically becomes an invariant of oriented 3-manifolds, provided it is unchanged by the relation $\leftrightarrow$.

We will describe such an invariant. It extends a similar result of Fukuhara [9] for the Kirby calculus.

Let $M^{3}=M(V, J)$ be the oriented 3-manifold with surgery data $V=V_{1} \cup \cdots \cup V_{n}, J=J_{1} \cup \cdots \cup J_{n}$. Consider the complementary fundamental group

$$
G=\pi_{1}\left(S^{3}-\stackrel{\circ}{V}\right)
$$

and the normal subgroup

$$
H=\left\{J_{1}, \ldots, J_{n}\right\}
$$

normally generated by the $J_{i}$ considered as loops in $S^{3}-\stackrel{\circ}{V}$. Note that the class of $J_{l}$ in $G$ is well defined up to conjugacy. Next define

$$
\bar{G}=G /[G, H], \quad \bar{H}=H /[G, H],
$$

where $[G, H]$ is the normal subgroup generated by commutators $g h g^{-1} h^{-1}$, $g \in G, h \in H$. Note that $[G, H] \triangleleft H$.

The group pair $(\bar{G}, \bar{H})$ may be called the surgery group pair, associated with the surgery data $(V, J)$. We shall call group pairs $(A, B)$ and $\left(A^{\prime} B^{\prime}\right)$ stably isomorphic if for some integers $m, n$, there is a commutative diagram

$$
\begin{gathered}
A \times \mathbf{Z}^{m} \cong A^{\prime} \times \mathbf{Z}^{n} \\
\text { (inclusion) } \times \text { id } \begin{array}{c}
\uparrow \\
B \times \mathbf{Z}^{m} \cong B^{\prime} \times \mathbf{Z}^{n} .
\end{array} \text { (inclusion) } \times \text { id }
\end{gathered}
$$

THEOREM 2. If $(V, J)$ and $\left(V^{\prime}, J^{\prime}\right)$ are surgery data in $S^{3}$ which produce orientation-preserving homeomorphic 3-manifolds, then their surgery group pairs are stably isomorphic.

This means that the stable isomorphism class of any surgery description of an $M^{3}$ depends only on $M$. This is at least as sensitive an invariant as the fundamental group of $M$ itself, noting the isomorphisms

$$
\bar{G} \times \mathbf{Z}^{m} / \bar{H} \times \mathbf{Z}^{m} \cong \bar{G} / \bar{H} \cong G / H \cong \pi_{1}(M) .
$$

Proof of Theorem 2, and related results, will appear in a forthcoming paper.

Added in proof. See [10]. The stable isomorphism class of $(\bar{G}, \bar{H})$ depends only on $\pi_{1}(M)$. 
Concluding remarks. Somewhat sharper versions of Theorem 1 may be obtained by closer inspection of the moves, I, II and III. We will say that the surgery data $(\hat{V}, \hat{J})$ is a trivial extension of $(V, J)$ if $V \subset \hat{V}$ and $J \subset \hat{J}$ and for each component $\hat{V}_{i}$ of $\hat{V}-V$, the curve $\hat{J}_{i}=\hat{J} \cap \hat{V}_{i}$ is a meridian of $\hat{V}_{i}$. In other words $(\hat{V}, \hat{J})$ is the result of a finite number of trivial insertions on $(V, J)$.

THEOREM 3. If $(V, J)$ and $\left(V^{\prime}, J^{\prime}\right)$ are surgery data in $S^{3}$, the following are equivalent:

(a) $M(V, J) \cong M\left(V^{\prime}, J^{\prime}\right)$ ( preserving orientation).

(b) There exist trivial extensions $(\hat{V}, \hat{J})$ and $\left(\hat{V}^{\prime}, \hat{J}^{\prime}\right)$ of $(V, J)$ and $\left(V^{\prime}, J^{\prime}\right)$, respectively, and an orientation preserving homeomorphism of pairs $\left(S^{3}\right.$-int $\left.\hat{V}, \hat{J}\right) \cong\left(S^{3}\right.$-int $\left.\hat{V}^{\prime}, \hat{J}^{\prime}\right)$.

(c) There exist trivial extensions of $(V, J)$ and $\left(V^{\prime}, J^{\prime}\right)$ which are related by a finite sequence of twists (II) and a single homeomorphism (I).

Proof. (c) $\Rightarrow$ (b) $\Rightarrow$ (a) is obvious. We argue (a) $\Rightarrow$ (c) as follows. If $M(V, J) \cong M\left(V^{\prime}, J^{\prime}\right)$, then $(V, J) \leftrightarrow\left(V^{\prime}, J^{\prime}\right)$ by Theorem 1 . The sequence of moves I, II, III may be altered so that all moves of type I occur together, so may be lumped together into a single homeomorphism, placed anywhere we like in the sequence of moves from $(V, J)$ to $\left(V^{\prime}, J^{\prime}\right)$. Also a twist on a particular component cannot occur before that component is inserted or after it is deleted. On the other hand, since different components are involved one may replace

$$
\left(V^{1}, J^{1}\right) \stackrel{\text { twist }}{\rightarrow}\left(V^{2}, J^{2}\right) \stackrel{\text { trivial insertion }}{\rightarrow}\left(V^{3}, J^{3}\right)
$$

by

$$
\left(V^{1}, J^{1}\right) \stackrel{\text { trivial insertion }}{\rightarrow}\left(V^{4}, J^{4}\right) \stackrel{\text { twist }}{\rightarrow}\left(V^{3}, J^{3}\right)
$$

So all the twists may be made to follow all the insertions. Likewise we can make them precede all the deletions, and we see that (a) $\Rightarrow(\mathrm{c})$.

Finally, we note that in the proof of Lemma 3 and in Fenn-Rourke's proof of Lemma 2, all the trivial insertions and deletions involve unknotted components. Call such an insertion (or deletion) doubly trivial.

THeORem 4. For surgery data, $(V, J) \leftrightarrow\left(V^{\prime}, J^{\prime}\right)$ if and only if they are related by a sequence of moves of types I, II and doubly trivial insertions and deletions. 


\section{REFERENCES}

[1] M. Dehn, Über die Topologie des drei-dimensionalen Räumes, Math. Ann., 75 (1910), 402-413.

[2] R. A. Fenn and C. Rourke, On Kirby's calculus of links, Topology, 18 (1979), 1-15.

[3] J. Hempel, Construction of Orientable 3-Manifolds, Topology of 3-manifolds (M. K. Fort, ed.), Prentice-Hall, N. J. 1962, 207-212.

[4] R. Kirby, A calculus for framed links in $S^{3}$, Invent. Math., 45 (1978), 35-56.

[5] W. B. R. Lickorish, A representation of orientable combinatorial 3-manifolds, Ann. Math., 76 (1962), 531-540.

[6] D. Rolfsen, Knots and Links, Publish or Perish, Berkeley 1976.

[7] H. Seifert, Topologie dreidimensionaler gefaserter Räume, Acta Math., 60 (1933), 147-238.

[8] A. Wallace, Modification and cobounding manifolds, Canad. J. Math., 12 (1960), 503-528.

[9] S. Fukuhara, On framed link groups, preprint.

[10] D. Rolfsen, A stable group pair invariant of 3-dimensional manifolds, Proc. Amer. Math. Soc. (to appear).

Received May 25, 1982.

UNIVERSITY OF BRITISH COLUMBIA

VANCOUVER, B.C. CANAdA V6T 1Y4 


\title{
PACIFIC JOURNAL OF MATHEMATICS EDITORS
}

\author{
DONALD BABBITT (Managing Editor) \\ University of California \\ Los Angeles, CA 90024 \\ Hugo Rossi \\ University of Utah \\ Salt Lake City, UT 84112 \\ C. C. MOORE and Arthur Ogus \\ University of California \\ Berkeley, CA 94720
}

\author{
J. Dugundir \\ Department of Mathematics \\ University of Southern California \\ Los Angeles, CA 90089-1113
}

R. FINN and H. SAMELSON

Stanford University

Stanford, CA 94305

ASSOCIATE EDITORS
R. ARENS
E. F. BECKENBACH
B. H. NeumanN
F. WOLF
K. YosHIDA (1906-1982)

\section{SUPPORTING INSTITUTIONS}

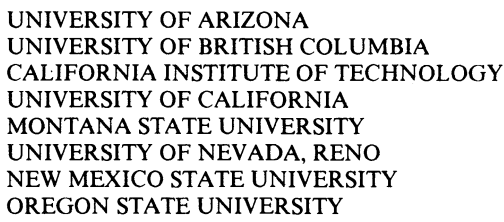

\author{
UNIVERSITY OF OREGON \\ UNIVERSITY OF SOUTHERN CALIFORNIA \\ STANFORD UNIVERSITY \\ UNIVERSITY OF HAWAII \\ UNIVERSITY OF TOKYO \\ UNIVERSITY OF UTAH \\ WASHINGTON STATE UNIVERSITY \\ UNIVERSITY OF WASHINGTON
}

The Supporting Institutions listed above contribute to the cost of publication of this Journal, but they are not owners or publishers and have no responsibility for its content or policies.

Mathematical papers intended for publication in the Pacific Journal of Mathematics should be in typed form or offset-reproduced (not dittoed), double spaced with large margins. Please do not use built up fractions in the text of the manuscript. However, you may use them in the displayed equations. Underline Greek letters in red, German in green, and script in blue. The first paragraph must be capable of being used separately as a synopsis of the entire paper. In particular it should contain no bibliographic references. Please propose a heading for the odd numbered pages of less than 35 characters. Manuscripts, in triplicate, may be sent to any one of the editors. Please classify according to the scheme of Math. Reviews, Index to Vol. 39. Supply name and address of author to whom proofs should be sent. All other communications should be addressed to the managing editor, or Elaine Barth, University of California, Los Angeles, California 90024.

There are page-charges associated with articles appearing in the Pacific Journal of Mathematics. These charges are expected to be paid by the author's University, Government Agency or Company. If the author or authors do not have access to such Institutional support these charges are waived. Single authors will receive 50 free reprints; joint authors will receive a total of 100 free reprints. Additional copies may be obtained at cost in multiples of 50 .

The Pacific Journal of Mathematics is issued monthly as of January 1966. Regular subscription rate: \$132.00 a year (6 Vol., 12 issues). Special rate: $\$ 66.00$ a year to individual members of supporting institutions.

Subscriptions, orders for numbers issued in the last three calendar years, and changes of address should be sent to Pacific Journal of Mathematics, P.O. Box 969, Carmel Valley, CA 93924, U.S.A. Old back numbers obtainable from Kraus Periodicals Co., Route 100, Millwood, NY 10546.

The Pacific Journal of Mathematics ISSN 0030-8730 is published monthly by the Pacific Journal of Mathematics at P.O. Box 969, Carmel Valley, CA 93924. Application to mail at Second-class postage rates is pending at Carmel Valley, California, and additional mailing offices. Postmaster: Send address changes to Pacific Journal of Mathematics, P. O. Box 969, Carmel Valley, CA 93924.

PUBLISHED BY PACIFIC JOURNAL OF MATHEMATICS, A NON-PROFIT CORPORATION

Copyright $(1984$ by Pacific Journal of Mathematics 


\section{Pacific Journal of Mathematics}

Vol. 110, No. $2 \quad$ October, 1984

Robert A. Bekes, The range of convolution operators $\ldots \ldots \ldots \ldots \ldots 257$

Dennis K. Burke and Sheldon Davis, Subsets of ${ }^{\omega} \omega$ and generalized metric

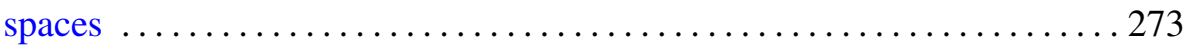

Giovanni Emmanuele, A remark on a paper: "Common fixed points of nonexpansive mappings by iteration" $\ldots \ldots \ldots \ldots \ldots \ldots \ldots \ldots \ldots 283$

I. Erdélyi and Sheng-Wang Wang, On strongly decomposable operators . . . 287

Gerhard Gierz, Injective Banach lattices with strong order units . . . . . . . 297

Maurizio Letizia, Quotients by complex conjugation of nonsingular quadrics and cubics in $\mathbf{P}_{\mathbf{C}}^{3}$ defined over $\mathbf{R} \ldots \ldots \ldots \ldots \ldots \ldots \ldots \ldots \ldots \ldots$

P. H. Maserick and Franciszek Hugon Szafraniec, Equivalent definitions

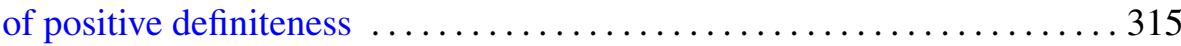

Costel Peligrad and S. Rubinstein, Maximal subalgebras of $C^{*}$-crossed

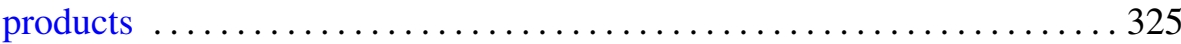

Derek W. Robinson and Sadayuki Yamamuro, Hereditary cones, order

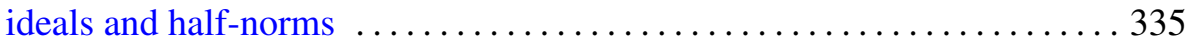

Derek W. Robinson and Sadayuki Yamamuro, The Jordan decomposition

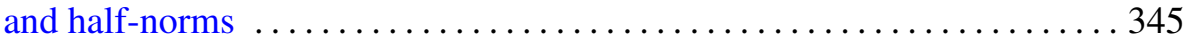

Richard Rochberg, Interpolation of Banach spaces and negatively curved

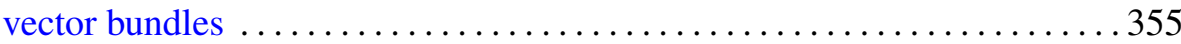

Dale Rolfsen, Rational surgery calculus: extension of Kirby's theorem 377

Walter Iaan Seaman, Helicoids of constant mean curvature and their Gauss maps

Diana Shelstad, Endoscopic groups and base change $\mathbf{C} / \mathbf{R}$

Jerrold Norman Siegel and Frank Williams, Numerical invariants of homotopies into spheres

Alladi Sitaram, Some remarks on measures on noncompact semisimple Lie groups

Teruhiko Soma, Atoroidal, irreducible 3-manifolds and 3-fold branched coverings of $S^{3}$

Jan de Vries, On the $G$-compactification of products

Hans Weber, Topological Boolean rings. Decomposition of finitely additive set functions 